Annals of Pure and Applied Mathematics

Vol. 16, No. 2, 2018, 353-263

ISSN: 2279-087X (P), 2279-0888(online)

Published on 23 February 2018

www.researchmathsci.org

DOI: http://dx.doi.org/10.22457/apam.v16n2a12

Annals of

Pure and Applied

Mathematics

\title{
Hall Effects on MHD Convective Rotating Flow of Through a Porous Mediumpast Infinite Vertical Plate
}

\author{
K.Raghunath ${ }^{1}$, R.Siva Prasad ${ }^{2}$ and G.S.S.Raju ${ }^{3}$ \\ ${ }^{1}$ JNTU College of Engineering, Anantapur, Andhra Pradesh, India \\ Email: kraghunath25@gmail.com \\ ${ }^{2}$ Department of Mathematics, S.K.University, Anantapur, Andhra Pradesh, India \\ Email: rsprasad_racharla@yahoo.co.in \\ ${ }^{3}$ Department of Mathematics, JNTUA College, Pulivendula, Andhra Pradesh, India \\ Email: rajugss@yahoo.com
}

Received 8 February 2018; accepted 18 February 2018

\begin{abstract}
In this paper, we have considered MHD natural convective rotating flow of a viscous, electrically conducting and incompressible fluid over an impulsively moving vertical plate embedded in porous medium and taking Hall current into account. The dimensionless governing coupled boundary layer equations are solved by regular perturbation technique. The effect of pertinent parameters on primary and secondary velocities, temperature and concentration for externally heating and cooling of the plate are shown graphically. Finally, the influence of non-dimensional parameters on the rate of heat and mass transfer and shear stress coefficient of the wall are in tabular forms.
\end{abstract}

Keywords: Heat and mass transfer, MHD flows, porous medium, unsteady flows and visco-elastic fluids.

\section{AMS Mathematics Subject Classification (2010): 35Q35}

\section{Introduction}

The study of natural convection flow induced by the simultaneous action of thermal and solutal buoyancy forces acting over bodies with different geometries in a fluid with porous medium is prevalent in many natural phenomena and has varied a wide range of industrial applications. For example, the presence of pure air or water is impossible because some foreign mass may be present either naturally or mixed with air or water due to industrial emissions, in atmospheric flows. Natural processes such as attenuation of toxic waste in water bodies, vaporization of mist and fog, photosynthesis, transpiration, sea-wind formation, drying of porous solids, and formation of ocean currents [1] occur due to thermal and solutal buoyancy forces developed as a result of difference in temperature or concentration or a combination of these two. Such configuration is also encountered in several practical systems for industry based applications viz. cooling of molten metals, heat exchanger devices, petroleum reservoirs, insulation systems, filtration, nuclear waste repositories, chemical catalytic reactors and processes, desert coolers, frost formation in vertical channels, wet bulb thermometers, etc. Considering the importance of such fluid flow problems, extensive and in-depth research works have been 


\section{K.Raghunath, R.Siva Prasad and G.S.S.Raju}

carried out by several researchers [2-10] in the past. Investigation of hydromagnetic natural convection flow with heat and mass transfer in porous and non-porous media has drawn considerable attentions of several researchers owing to its applications in geophysics, astrophysics, aeronautics, meteorology, electronics, chemical, and metallurgy and petroleum industries. Magnetohydrodynamic (MHD) natural convection flow of an electrically conducting fluid with porous medium has also been successfully exploited in crystal formation. Recently Raghunath and Siva Prasad [11] discussed heat and mass transfer on unsteady MHD flow of a visco elastic fluid past an infinite vertical oscillating porous plate. Oreper and Szekely [12] have found that the presence of a magnetic field can suppress natural convection currents and the strength of magnetic field is one of the important factors in reducing non-uniform composition thereby enhancing quality of the crystal. In addition to it, the thermal physics of hydromagnetic problems with mass transfer is of much significance in MHD flow-meters, MHD energy generators, MHD pumps, controlled thermo-nuclear reactors, MHD accelerators, etc. Keeping in view the importance of such study, Hossain and Mandal [13] investigated mass transfer effects on unsteady hydromagnetic free convection flow past an accelerated vertical porous plate. Recently, Veera Krishna and Reddy [14] discussed MHD free convective rotating flow of visco-elastic fluid past an infinite vertical oscillating plate.

\section{Formulation and solution of the problem}

Consider an unsteady MHD natural convective flow with heat and mass transfer of an optically thick radiating, incompressible and electrically conducting viscous fluid past an infinite vertical plate is embedded in a uniform porous medium with a rotating system taking hall current into account. Consider $x^{\prime}$-axis is along the plate in upward direction and $y^{\prime}$-axis is normal to plane of the plate in the fluid. A uniform transverse magnetic field $B_{0}$ is applied in a direction which is parallel to $y^{\prime}$-axis. The fluid and plate rotate with uniform angular velocity $\Omega^{\prime}$ about the $y^{\prime}$-axis. Initially i.e. at time $t^{\prime} \leq 0$, both the fluid and plate or in rest and these are maintained at a uniform temperature $T_{\infty}^{\prime}$. Also species concentration is at the surface of the plate as well as at every point within the fluid and it is maintained at uniform concentration $C_{\infty}^{\prime}$. At time $t^{\prime} \geq 0$, plate starts moving in $x^{\prime}$-direction with uniform velocity $U_{0}$ in its own plane. The temperature of the plate is raised or lowered to $T_{\infty}^{\prime}+\left(T_{w}^{\prime}-T_{\infty}^{\prime}\right) t^{\prime} / t_{0}$ when $0 \leq t^{\prime} \leq t_{0}$, and it is maintained at uniform temperature $T_{w}^{\prime}$ when $t^{\prime} \geq t_{0}$.

Also, at time $t^{\prime} \geq 0$, species concentration is at the surface of the plate, it is raised to uniform species concentration $C_{w}^{\prime}$ and it is maintained thereafter. Geometry of the problem is show in Fig.1. Since plate is an infinite extent in $x^{\prime}$ and $z^{\prime}$ directions and it is electrically non-conducting, all physical quantities except pressure depend on $y^{\prime}$ and $t^{\prime}$ only. Also, no applied or polarized voltages are assumed to exist, so that the effect of polarization of fluid is negligible. The induced magnetic field also is neglected. The magnetic Reynolds number is very small for liquid metals and partially ionized fluids which are commonly used in industrial applications. 
Hall Effects on MHD Convective Rotating Flow of Through a Porous Mediumpast Infinite Vertical Plate

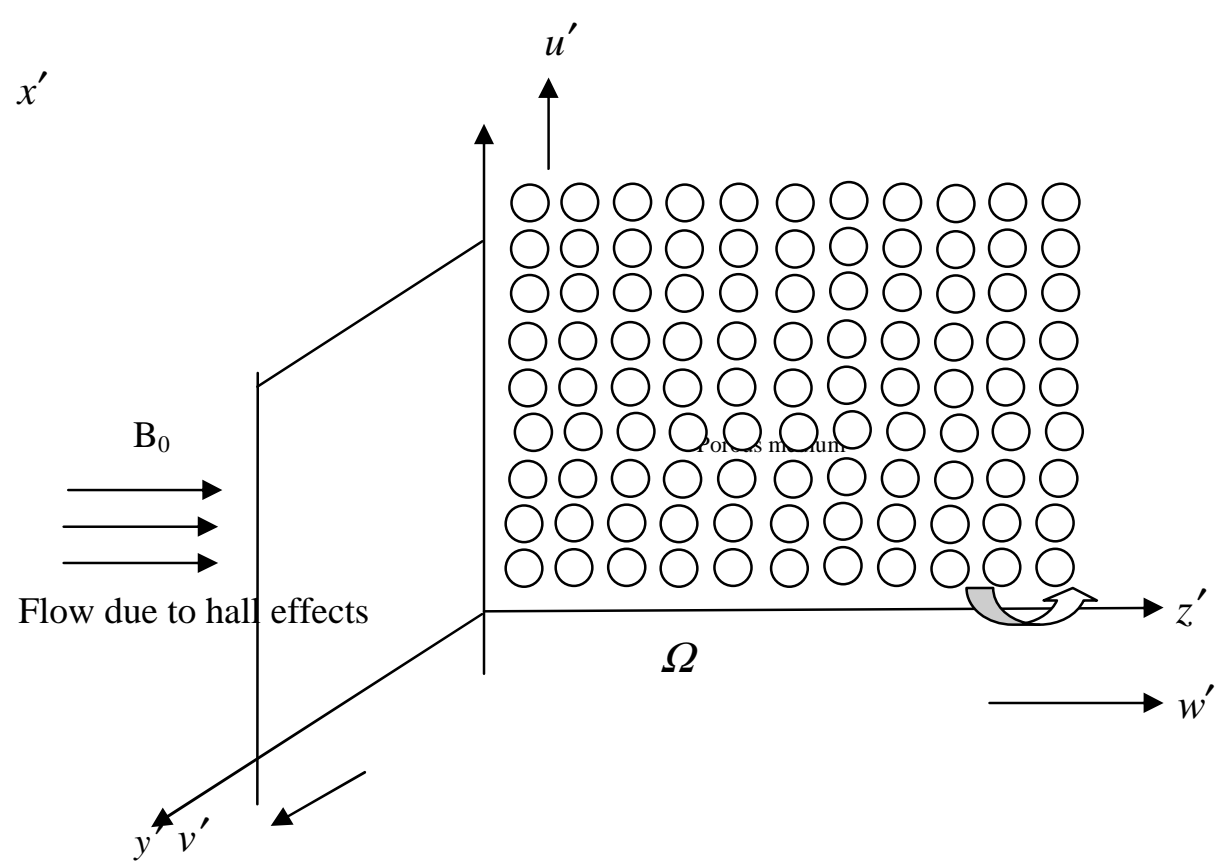

Figure 1: Physical configuration of the problem

Keeping in view of these assumptions and under the Boussinesq's approximation, the governing equations are given by

$$
\begin{aligned}
& \frac{\partial u^{\prime}}{\partial t^{\prime}}-2 \Omega^{\prime} v^{\prime}=v \frac{\partial^{2} u^{\prime}}{\partial z^{\prime 2}}+\frac{B_{0} J_{y}}{\rho}-\frac{v u^{\prime}}{k_{1}}+g \beta\left(T^{\prime}-T_{\infty}^{\prime}\right)+g \beta^{*}\left(C^{\prime}-C_{\infty}^{\prime}\right) \\
& \frac{\partial v^{\prime}}{\partial t^{\prime}}-2 \Omega^{\prime} u^{\prime}=v \frac{\partial^{2} v^{\prime}}{\partial z^{\prime 2}}-\frac{B_{0} J_{x}}{\rho}-\frac{v}{k_{1}} v^{\prime} \\
& \frac{\partial T^{\prime}}{\partial t^{\prime}}=\frac{k}{\rho C_{p}} \frac{\partial^{2} T^{\prime}}{\partial z^{\prime 2}}-\frac{1}{\rho C_{p}} \frac{\partial q_{r}}{\partial z^{\prime}}+\frac{D_{m} k_{T}}{C_{s} C_{p}} \frac{\partial^{2} C^{\prime}}{\partial z^{\prime 2}} \\
& \frac{\partial C^{\prime}}{\partial t^{\prime}}=D \frac{\partial^{2} C^{\prime}}{\partial z^{\prime 2}}+\frac{D_{m} k_{T}}{T_{m}} \frac{\partial^{2} T^{\prime}}{\partial z^{\prime 2}}
\end{aligned}
$$

The boundary conditions taken for the problem are

$$
\begin{aligned}
& U^{\prime}=u_{p}^{\prime}, T=T_{\infty}^{\prime}+\varepsilon\left(T_{w}^{\prime}-T_{\infty}^{\prime}\right) e^{n^{\prime} t^{\prime}}, C=C_{\infty}^{\prime}+\varepsilon\left(C_{w}^{\prime}-C_{\infty}^{\prime}\right) e^{n^{\prime t^{\prime}}} \text { at } z^{\prime}=0, \\
& U^{\prime} \rightarrow 0, \theta \rightarrow \theta_{\infty}, \phi \rightarrow \phi_{\infty} \quad \text { at } \quad z^{\prime} \rightarrow \infty
\end{aligned}
$$

The radiative flux term by using the Rosseland approximation is given by

$$
q_{r}^{\prime}=-\frac{4 \sigma^{*}}{3 k^{*}}\left(\frac{\partial T^{\prime 4}}{\partial z^{\prime}}\right)_{z=0}
$$




\section{K.Raghunath, R.Siva Prasad and G.S.S.Raju}

Using the Rosseland approximation, present analysis is limited to optically thick fluids. If temperature differences within the flow are sufficiently very small then eq.(6) can be linearised by expanding $T^{\prime}$ into the taylor series about $T_{\infty}^{\prime}$ which after neglecting higher order terms the form

$$
T^{\prime 4} \cong T_{\infty}^{\prime 3}-3 T_{\infty}^{\prime 4}
$$

Substituting equation (6) and (7) into equation (3) we obtain

$$
\frac{\partial T^{\prime}}{\partial t^{\prime}}=\frac{k}{\rho C_{p}} \frac{\partial^{2} T^{\prime}}{\partial z^{\prime 2}}+\frac{1}{\rho C_{p}} \frac{16 \sigma^{*} T_{\infty}^{\prime 3}}{3 k^{*}} \frac{\partial^{2} T^{\prime}}{\partial z^{\prime 2}}+\frac{D_{m} k_{T}}{C_{s} C_{p}} \frac{\partial^{2} C^{\prime}}{\partial z^{\prime 2}}
$$

When the strength of the magnetic field is very large, the generalized ohm's law is modified to include the hall current so that

$$
J+\frac{\omega_{e} \tau_{e}}{B_{O}}(J \times B)=\sigma\left[E+V \times B+\frac{1}{e \eta_{e}} \nabla P_{e}\right]
$$

The ion-slip and thermo electric effects are not included in equation (9). Further it is assumed that $\omega_{e} \tau_{e} \sim 0$ (1) and $\omega_{i} \tau_{i}<<1$, In the equation (9) the electron pressure gradient, the ion-slip and thermo-electric effects are neglected. We also assume that the electric field $E=0$ under assumptions reduces to

$$
\begin{gathered}
J_{x}+m J_{y}=\sigma B_{0} v \\
J_{y}-m J_{x}=-\sigma B_{0} u
\end{gathered}
$$

where $m=\tau_{\mathrm{e}} \omega_{e}$ is the hall parameter.

On solving equations (10) and (11) we obtain

$$
\begin{aligned}
& J_{x}=\frac{\sigma B_{0}}{1+m^{2}}(v+m u) \\
& J_{y}=\frac{\sigma B_{0}}{1+m^{2}}(m v-u)
\end{aligned}
$$

Substituting the equations (12) and (13) in (2) and (1) respectively, we obtain

$$
\begin{aligned}
& \frac{\partial u^{\prime}}{\partial t^{\prime}}-2 \Omega^{\prime} v^{\prime}=v \frac{\partial^{2} u^{\prime}}{\partial z^{\prime 2}}+\left(\frac{\sigma B_{0}^{2}}{1+m^{2}}(m v-u)-\frac{v}{k}\right) u^{\prime}+g \beta\left(T^{\prime}-T_{\infty}^{\prime}\right)+g \beta^{*}\left(C^{\prime}-C_{\infty}^{\prime}\right) \\
& \frac{\partial v^{\prime}}{\partial t^{\prime}}-2 \Omega^{\prime} u^{\prime}=v \frac{\partial^{2} v^{\prime}}{\partial z^{\prime 2}}-\left(\frac{\sigma B_{0}^{2}}{1+m^{2}}(v+m u)+\frac{v}{k}\right) v^{\prime}-\frac{v}{k_{1}} v^{\prime}
\end{aligned}
$$

Introducing the following non-dimensional quantities are,

$$
\begin{aligned}
& u=\frac{u^{\prime}}{U_{0}}, v=\frac{v^{\prime}}{U_{0}}, z=\frac{z^{\prime} U_{0}^{2}}{v}, t=\frac{t^{\prime} U_{0}^{2}}{v}, \theta^{*}=\frac{T^{\prime}-T_{\infty}^{\prime}}{T_{w}^{\prime}-T_{\infty}^{\prime}}, \phi^{*}=\frac{C^{\prime}-C_{\infty}^{\prime}}{C_{w}^{\prime}-C_{\infty}^{\prime}}, \\
& M^{2}=\frac{\sigma B_{0}^{2} v}{\rho U_{0}^{2}}, \Omega=\frac{v \Omega^{\prime}}{U_{0}{ }^{2}}, K=\frac{k_{1} U_{0}^{2}}{v^{2}}, \mathrm{Gr}=\frac{g \beta v\left(T_{w}^{\prime}-T_{\infty}^{\prime}\right)}{U_{0}^{3}}, \mathrm{Gm}=\frac{g \beta^{*} v\left(C_{w}^{\prime}-C_{\infty}^{\prime}\right)}{U_{0}^{3}},
\end{aligned}
$$


Hall Effects on MHD Convective Rotating Flow of Through a Porous Mediumpast Infinite Vertical Plate

$$
\operatorname{Pr}=\frac{v_{p} \phi_{p}}{k}, N=\frac{16 \sigma^{*} T_{\infty}^{3}}{3 k k^{*}}, \mathrm{Sc}=\frac{v}{D}, \mathrm{Sr}=\frac{D_{m} k_{T}\left(T_{w}^{\prime}-T_{\infty}^{\prime}\right)}{v T_{m}\left(C_{w}^{\prime}-C_{\infty}^{\prime}\right)}
$$

Then the resultant non-dimensional equations are

$$
\begin{aligned}
& \frac{\partial u}{\partial t}+2 \Omega v=\frac{\partial^{2} u}{\partial z^{2}}-\frac{M^{2}(m v-u)}{1+m^{2}} u-\frac{u}{k_{1}}+\operatorname{Gr} \theta+\operatorname{Gm} \phi \\
& \frac{\partial v}{\partial t}-2 \Omega u=\frac{\partial^{2} v}{\partial z^{2}}-\frac{M^{2}(v+m u)}{1+m^{2}}-\frac{v}{k_{1}} \\
& \frac{\partial \theta}{\partial t}=\left(\frac{1+N}{P_{r}}\right) \frac{\partial^{2} \theta}{\partial z^{2}}+E c\left(\frac{\partial q}{\partial z}\right)^{2} \\
& \frac{\partial \phi}{\partial t}=\frac{1}{\operatorname{Sc}} \frac{\partial^{2} \phi}{\partial z^{2}}+\operatorname{Sr} \frac{\partial^{2} \theta}{\partial z^{2}}
\end{aligned}
$$

Combining equation (16) and (17), let $q=u+i v$, we obtain

$$
\begin{gathered}
\frac{\partial q}{\partial t}=\frac{\partial^{2} q}{\partial z^{2}}-\lambda q+\operatorname{Gr} \theta+\operatorname{Gm} \phi \\
\text { where, } \lambda=\frac{M^{2}}{1+m^{2}}+2 i E^{-1}+\frac{1}{K}
\end{gathered}
$$

With the following dimensionless boundary conditions

$$
\begin{array}{ll}
q=U_{0}, \theta=1+\varepsilon e^{n t}, \phi=1+\varepsilon e^{n t} & \text { at } \quad z=0 \\
q \rightarrow 0, \theta \rightarrow 0, \phi \rightarrow 0 & \text { at } \quad z \rightarrow \infty
\end{array}
$$

We use the linear transformation for low value of $\varepsilon$ to solve the equations (18)-(20) subject to the boundary conditions (21) and (22) as follows

$$
\begin{aligned}
& q(z, t)=q_{0}(z)+\varepsilon e^{i n t} q_{1}(z)+O\left(\varepsilon^{2}\right) \\
& \theta(z, t)=\theta_{0}(z)+\varepsilon e^{i n t} \theta_{1}(z)+O\left(\varepsilon^{2}\right) \\
& \phi(z, t)=\phi_{0}(z)+\varepsilon e^{i n t} \phi_{1}(z)+O\left(\varepsilon^{2}\right)
\end{aligned}
$$

After substituting the equation (23) to (25) into (18) to (20) we have

$$
\begin{aligned}
& \frac{\partial^{2} q_{0}}{\partial z^{2}}-\lambda q_{0}=-\operatorname{Gr} \theta_{0}-\operatorname{Gm} \phi_{0} \\
& \frac{\partial^{2} q_{1}}{\partial z^{2}}-\lambda q_{1}-i n q_{1}=-\operatorname{Gr} \theta_{1}-\operatorname{Gm} \phi_{1} \\
& R \frac{\partial^{2} \theta_{0}}{\partial z^{2}}=-E c\left(\frac{\partial q_{0}}{\partial z}\right)^{2} \\
& R \frac{\partial^{2} \theta_{1}}{\partial z^{2}}-i n \theta_{1}=-2 E c \frac{\partial q_{0}}{\partial z} \frac{\partial q_{1}}{\partial z}
\end{aligned}
$$


K.Raghunath, R.Siva Prasad and G.S.S.Raju

$$
\begin{aligned}
& \frac{1}{\operatorname{Sc}} \frac{\partial^{2} \phi_{0}}{\partial z^{2}}=-\operatorname{Sr} \frac{\partial^{2} \theta_{0}}{\partial z^{2}} \\
& \frac{1}{\operatorname{Sc}} \frac{\partial^{2} \phi_{1}}{\partial z^{2}}-i n \phi_{1}=-\operatorname{Sr} \frac{\partial^{2} \theta_{1}}{\partial z^{2}}
\end{aligned}
$$

With the following boundary equations

$$
\begin{aligned}
& q_{0}=U_{p}, q_{1}=0, \theta_{0}=1, \theta_{1}=1, \phi_{0}=1, \phi_{1}=1 \quad \text { at } z=0, \\
& q_{0}=q_{1}=\theta_{0}=\theta_{1}=\phi_{0}=\phi_{1}=0 \quad \text { at } \quad z \rightarrow \infty
\end{aligned}
$$

Assuming the viscous dissipation parameter (Ecket number $E c$ ) is small, we can solve the non linear coupled equations (26)-(31) writing the asymptotic expansion as follows:

$$
\begin{aligned}
& q_{0}(z)=q_{01}(z)+E c q_{02}(z)+O\left(E c^{2}\right) \\
& q_{1}(z)=q_{11}(z)+E c q_{12}(z)+O\left(E c^{2}\right) \\
& \theta_{0}(z)=\theta_{01}(z)+E c \theta_{02}(z)+O\left(E c^{2}\right) \\
& \theta_{1}(z)=\theta_{11}(z)+E c \theta_{12}(z)+O\left(E c^{2}\right) \\
& \phi_{0}(z)=\phi_{01}(z)+E c \phi_{02}(z)+O\left(E c^{2}\right) \\
& \phi_{1}(z)=\phi_{11}(z)+E c \phi_{12}(z)+O\left(E c^{2}\right)
\end{aligned}
$$

Now substituting equation (34) to (39) into equations (26) to (31), we obtain the following sequence of approximation for $O(E c)$ :

$$
\begin{aligned}
& \frac{\partial^{2} q_{01}}{\partial z^{2}}-\lambda q_{01}=-\operatorname{Gr} \theta_{01}-\operatorname{Gm} \phi_{01} \\
& \frac{\partial^{2} q_{02}}{\partial z^{2}}-\lambda q_{02}=-\operatorname{Gr} \theta_{02}-\operatorname{Gm} \phi_{02} \\
& \frac{\partial^{2} q_{11}}{\partial z^{2}}-\lambda q_{11}-i n q_{11}=-\operatorname{Gr} \theta_{11}-\operatorname{Gm} \phi_{11} \\
& \frac{\partial^{2} q_{12}}{\partial z^{2}}-\lambda q_{12}-i n q_{12}=-\operatorname{Gr} \theta_{12}-\operatorname{Gm} \phi_{12} \\
& R \frac{\partial^{2} \theta_{01}}{\partial z^{2}}=0 \\
& R \frac{\partial^{2} \theta_{02}}{\partial z^{2}}=-\left(\frac{\partial q_{01}}{\partial z}\right)^{2} \\
& R \frac{\partial^{2} \theta_{11}}{\partial z^{2}}-i n \theta_{11}=0 \\
& R \frac{\partial^{2} \theta_{12}}{\partial z^{2}}-i n \theta_{12}=-2 \frac{\partial q_{01}}{\partial z} \frac{\partial q_{11}}{\partial z} \\
& \frac{1}{\mathrm{Sc}} \frac{\partial^{2} \phi_{01}}{\partial z^{2}}=-\operatorname{Sr} \frac{\partial^{2} \theta_{01}}{\partial z^{2}} \\
& \frac{1}{\mathrm{Sc}} \frac{\partial^{2} \phi_{02}}{\partial z^{2}}=-\operatorname{Sr} \frac{\partial^{2} \theta_{02}}{\partial z^{2}}
\end{aligned}
$$


Hall Effects on MHD Convective Rotating Flow of Through a Porous Mediumpast Infinite Vertical Plate

$$
\begin{aligned}
& \frac{1}{\operatorname{Sc}} \frac{\partial^{2} \phi_{11}}{\partial z^{2}}-i n \phi_{11}=-\operatorname{Sr} \frac{\partial^{2} \theta_{11}}{\partial z^{2}} \\
& \frac{1}{\operatorname{Sc}} \frac{\partial^{2} \phi_{12}}{\partial z^{2}}-i n \phi_{12}=-\operatorname{Sr} \frac{\partial^{2} \theta_{12}}{\partial z^{2}}
\end{aligned}
$$

Subject to the following boundary conditions

$$
\begin{aligned}
& q_{01}=U_{0}, q_{11}=0, \theta_{01}=\theta_{11}=\phi_{01}=\phi_{11}=1, q_{02}=q_{12}=\theta_{02}=\theta_{12}=\phi_{02}=\phi_{12}=0 \text { at } z=0(52) \\
& q_{01}=q_{11}=\theta_{01}=\theta_{11}=\phi_{01}=\phi_{11}=q_{02}=q_{12}=\theta_{02}=\theta_{12}=\phi_{02}=\phi_{12}=0 \quad \text { at } z \rightarrow \infty
\end{aligned}
$$

Now solving the linear equations (40)-(51) with the boundary conditions (52) (53) we get the results as follows

$$
\begin{aligned}
& q=a_{2} e^{-h_{1} z}+a_{3}+E c\left(a_{10} e^{-h_{1} z}+a_{11} e^{-2 h_{1} z}+a_{12}\right)+\varepsilon e^{i n t}\left\{-\left(a_{4}+a_{5}+a_{6}\right) e^{-h_{2} z}+\left(a_{4}+a_{6}\right) e^{-h_{3} z}+\right. \\
& +a_{5} e^{-h_{4} z}+E c\left[-\left(m_{8}+m_{9}+m_{10}+m_{11}+m_{12}\right) e^{-h_{2} z}+m_{8} e^{-h_{3} z}+m_{9} e^{-\left(h_{1}+h_{2}\right) z}+\right. \\
& \left.\left.+m_{10} e^{-\left(h_{1}+h_{3}\right) z}+m_{11} e^{-\left(h_{1}+h_{4}\right) z}+m_{12} e^{-h_{4} z}\right]\right\} \\
& \theta=1+E c a_{7}\left(e^{-2 h_{1} z}-1\right)+\varepsilon e^{i n t}\left\{e^{-h_{3} z}+E c\left[\left(-m_{1}+m_{2}+m_{3}\right) e^{-h_{3} z}\right.\right. \\
& \left.\left.+m_{1} e^{-\left(h_{1}+h_{2}\right) z}-m_{2} e^{-\left(h_{1}+h_{3}\right) z}-m_{3} e^{-\left(h_{1}+h_{4}\right) z}\right]\right\} \\
& \phi=1+E c a_{8}\left(1-e^{-2 h_{1} z}\right)+\varepsilon e^{i n t}\left\{\left(1+a_{1}\right) e^{-h_{4} z}-a_{1} e^{-h_{3} z}+E c\left[\left(m_{4}+m_{5}-m_{6}-m_{7}\right) e^{-h_{4} z}\right.\right. \\
& \left.\left.-m_{4} e^{-h_{3} z}-m_{5} e^{-\left(h_{1}+h_{2}\right) z}+m_{6} e^{-\left(h_{1}+h_{3}\right) z}+m_{7} e^{-\left(h_{1}+h_{4}\right) z}\right]\right\}
\end{aligned}
$$

Some important physical quantities related to the heat and mass transfer characteristics are the skin friction coefficient, Nusselt number and Sherwood numberare given by

$$
\begin{aligned}
& \tau=\left(\frac{\partial q}{\partial z}\right)_{z=0} \\
& \tau=-a_{2} h_{1}-E c\left(a_{10} h_{1}+2 a_{11} h_{1}\right)-\varepsilon e^{i n t}\left\{-\left(a_{4}+a_{5}+a_{6}\right) h_{2}+\left(a_{4}+a_{6}\right) h_{3}+a_{5} h_{4}+E c\left[-\left(m_{8}+m_{9}+m_{10}\right.\right.\right. \\
& \left.\left.\left.+m_{11}+m_{12}\right) h_{2}+m_{8} h_{3}+m_{9}\left(h_{1}+h_{2}\right)+m_{10}\left(h_{1}+h_{3}\right)+m_{11}\left(h_{1}+h_{4}\right)+m_{12} h_{4}\right]\right\} \\
& N u=\left(\frac{\partial \theta}{\partial z}\right)_{z=0} \\
& =-2 E c a_{7} h_{1}-\varepsilon e^{i n t}\left\{h_{3}+E c\left[\left(-m_{1}+m_{2}+m_{3}\right) h_{3}+m_{1}\left(h_{1}+h_{2}\right)-m_{2}\left(h_{1}+h_{3}\right)-m_{3}\left(h_{1}+h_{4}\right)\right]\right\} \\
& S h=\left(\frac{\partial \phi}{\partial z}\right)_{z=0} \\
& S h=2 E c a_{8} h_{1}-\varepsilon e^{i n t}\left\{\left(1+a_{1}\right) h_{4}-a_{1} h_{3}+E c\left[\left(m_{4}+m_{5}-m_{6}-m_{7}\right) h_{4}\right.\right. \\
& \left.\left.-m_{4} h_{3}-m_{5}\left(h_{1}+h_{2}\right)+m_{6}\left(h_{1}+h_{3}\right)+m_{7}\left(h_{1}+h_{4}\right)\right]\right\}
\end{aligned}
$$


K.Raghunath, R.Siva Prasad and G.S.S.Raju

\section{Results and discussion}

We have considered the unsteady free convective flow of a viscous incompressible electrically conducting fluid over an infinite vertical porous plate under the influence of uniform transverse magnate field taking hall current into account. The governing equations of the flow field are solved by a regular perturbation method for small Eckert number $E c$. The closed form solutions for the velocity, temperature and concentration have been derived analytically and also its behavior is computationally discussed with reference to different flow parameters like $M$ Hartmann number, $E$ Ekman number, $K$ porosity parameter, $m$ hall parameter, $\mathrm{Gr}$ the thermal Grashof number, $\mathrm{Gm}$ the mass Grashof number, $N$ Radiation parameter, Pr is the Prandtl number, Sr Soret parameter, Sc is the Schmidt number and time $t$. Figures (2-5) represent velocity, Figures (6) and Figures (7) represent the temperature and concentration distributions respectively. The stresses, Nusselt number and Sherwood number at the plate are evaluated numerically and discussed with governing parameters and are tabulated in the tables (1-3). Fixing the parameters $n=1$ and $E c=0.01$.

From the Figures (2), we noticed that the magnitude of the velocity components $u$ and $v$ as well as resultant velocity are reduces with increasing the intensity of the magnetic field $M$. This is because of the reason that effects of a transverse magnetic field on an electrically conducting fluid gives rise to a resistive type force (called Lorentz force) similar to drag force and upon increasing the values of $M$ increases the drag force which has tendency to slow down the motion of the fluid. Both the magnitude of the velocity components $u$ and $v$ are enhances with increasing Ekman number E due to the rotation of the plate. The resultant velocity is also experiences enhancement throughout the fluid region with increasing $E$ (Figures 3 ).

In fluid dynamics, flow through porous media, the Darcy number (Da) represents the relative effect of the permeability of the medium versus its cross-sectional area commonly the diameter squared. The number is named after Henry Darcy and is found from nondimensionalizing the differential form of Darcy's Law. This number should not be confused with the Darcy friction factor which applies to pressure drop in a pipe. The magnitude of both the velocity components enhances with increasing the permeability parameter D. Also we observe that lower the permeability of the porous medium lesser the fluid speed in the entire fluid region. From the figure (4) depicts that the velocity component $u$ increases and $v$ reduces and the resultant velocity also enhances with increasing permeability of the porous medium (Darcy parameter). Lower the permeability of the porous medium lesser the fluid speed in the entire region. We noticed that from the figures (5) the magnitude of the velocity component $u$ increases and $v$ initially enhances and then gradually reduces throughout the fluid region with increasing thermal Grashof number Gr or mass Grashof number Gm. The resultant velocity is also boost up throughout the fluid medium with increasing Gr or Gm. Figures (6) showed the effect of Radiation parameter $N$ and the Prandtl number Pr on the temperature of the flow field. With increasing radiation parameter $N$ reduces the temperature of the flow field. This may happen due the elastic property of the fluid. We also noted that the temperature of the flow field diminishes as the Prandtl number increases. This is consistent with the fact that the thermal boundary layer thickness decreases with increasing Prandtl number. 
Hall Effects on MHD Convective Rotating Flow of Through a Porous Mediumpast Infinite Vertical Plate

Figures (7) depict the effect of the Soret number Sr and Schmidt number Sc on the concentration distribution. It is observed that presence of the Soret parameter $\mathrm{Sr}$ increase the concentration distribution. The concentration distribution linearly decreases at all points of the flow field with the increase in the Schmidt number Sc.
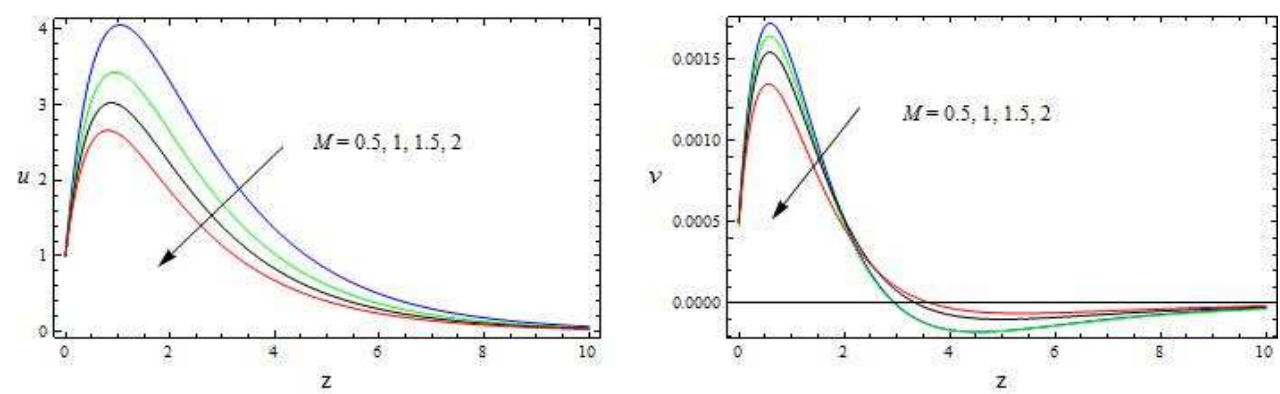

Figures 2: The velocity profiles for $u$ and $v$ with $M$

$E=0.1, K=0.5, \mathrm{Gr}=3, \mathrm{Gm}=1, \mathrm{Pr}=0.71, N=0.3, \mathrm{Sr}=0.5, \mathrm{Sc}=0.22, t=0.1$
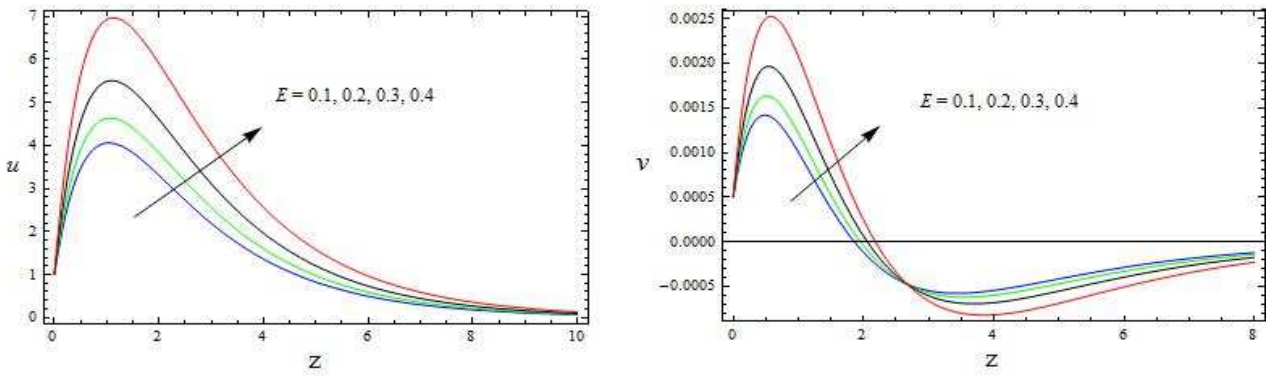

Figures 3: The velocity profiles for $u$ and $v$ with $E$ $M=0.5, K=0.5, \mathrm{Gr}=3, \mathrm{Gm}=1, \mathrm{Pr}=0.71, N=0.3, \mathrm{Sr}=0.5, \mathrm{Sc}=0.22, t=0.1$
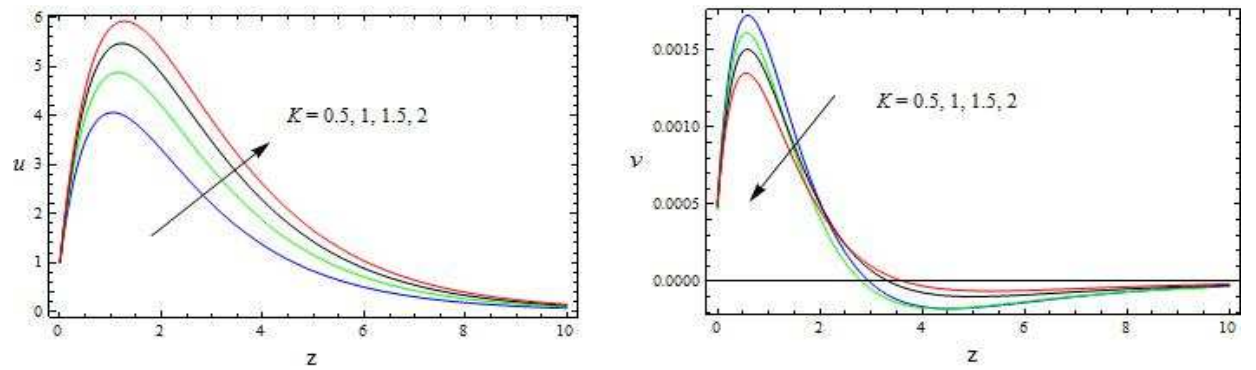

Figure 4: The velocity Profiles for $u$ and $v$ with $K$ $M=0.5, E=0.1, \mathrm{Gr}=3, \mathrm{Gm}=1, \mathrm{Pr}=0.71, N=0.3, \mathrm{Sr}=0.5, \mathrm{Sc}=0.22, t=0.1$ 
K.Raghunath, R.Siva Prasad and G.S.S.Raju
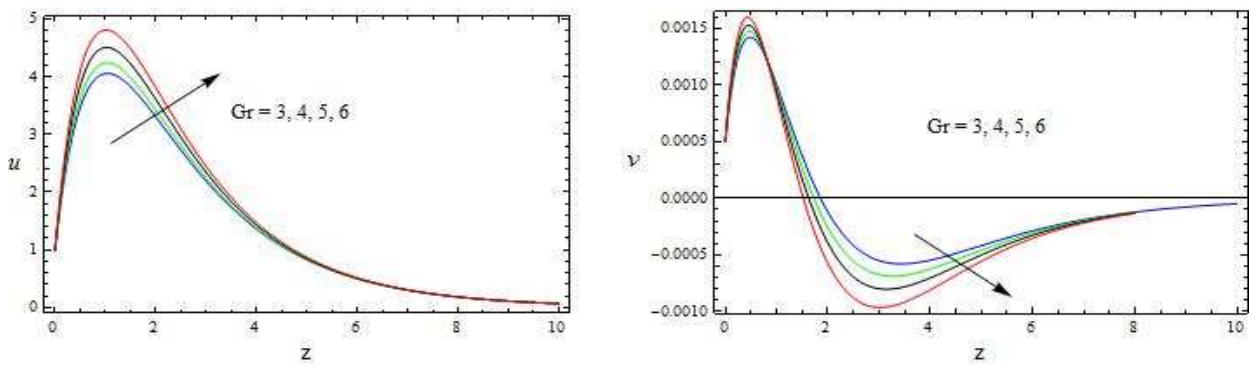

Figure 5: The velocity profiles for $u$ and $v$ with Gr

$M=0.5, E=0.1, K=0.5, \mathrm{Gm}=1, \mathrm{Pr}=0.71, N=0.3, \mathrm{Sr}=0.5, \mathrm{Sc}=0.22, t=0.1$
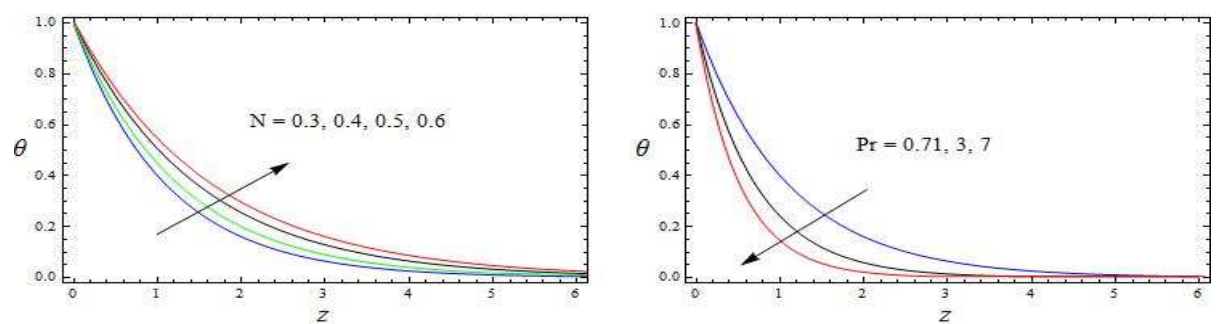

Figure 6: Temperature profiles with $N(\operatorname{Pr}=0.71)$ and $\operatorname{Pr}(N=0.3)$
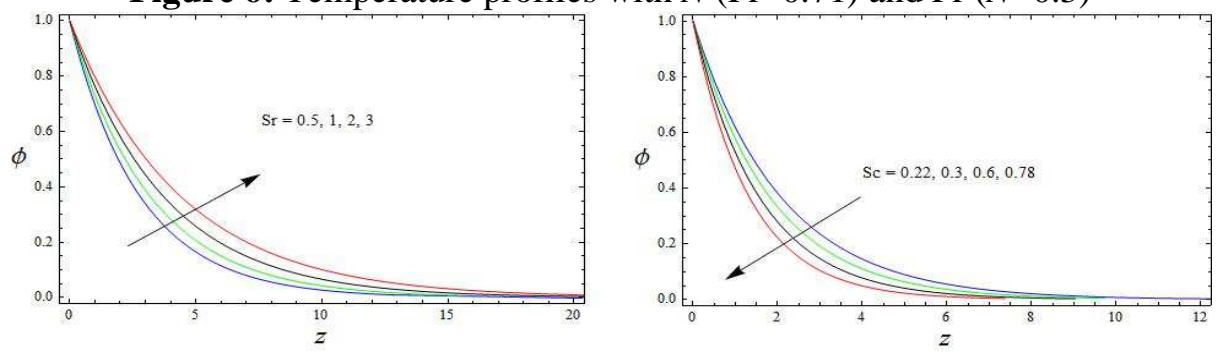

Figure 7: Concentration Profiles with $\mathrm{Sr}(\mathrm{Sc}=0.22)$ and $\mathrm{Sc}(\mathrm{Sr}=0.5)$

\section{Conclusions}

1. The rotation parameter increases the velocity close to the wall but there have been a decrease in velocity outlying from the wall.

2. The velocity increases with increase in $K, N, \mathrm{Gr}, \mathrm{Gm}, \mathrm{Sr}, m$ and $t$.

3. The resultant velocity diminishes with increasing $M$ or Sc or Pr.

4. The temperature reduces with increase in the value of Prandtl number and increases with Radiation parameter.

5. In presence of $\mathrm{Sr}$ increase the concentration. It linearly decreases at all points of the flow field with increase in Sc.

6. The skin friction coefficients both $\tau_{x y}$ or $\tau_{x z}$ increase with the increasing in $E$, $\mathrm{Gr}$ and $\mathrm{Sr}$ and also reduce with increasing $K, m$ and $\mathrm{Gm}$. The component $\tau_{x y}$ 
Hall Effects on MHD Convective Rotating Flow of Through a Porous Mediumpast Infinite Vertical Plate

enhances and $\tau_{x z}$ reduces with increasing $M$ and Sc. The reversal behaviour is observed with increasing Radiation parameter or Pr.

7. Nusselt number $(\mathrm{Nu})$ reduces with increase radiation parameter. Also enhance the rate of heat transfer with increase Prandtl number or the frequency parameter.

8. The rate of mass transfer increases with increasing of Schmidt number and in the presence of Soret number decreases Sherwood number.

\section{REFERENCES}

1. A.Bejan, Convection heat transfer, 2nd ed, New York, Wiley, 1993.

2. B.Gebhart and L.Pera, The nature of vertical natural convection flows resulting from the combined buoyancy effects of thermal and mass diffusion, Int J Heat Mass Transfer, 14(12) (1971) 2025-2050.

3. A.A.Raptis, Free convection and mass transfer effects on the oscillatory flow past an infinite moving vertical isothermal plate with constant suction and heat sources. Astro Phys Space Sci., 86(1) (1982) 43-53.

4. A.Bejan and K.R.Khair, Heat and mass transfer by natural convection in a porous medium, Int J Heat Mass Transfer, 28(5) (1985) 909-918.

5. J.Y.Jang and W.J.Chang, Buoyancy-induced inclined boundary layer flow in a porous medium resulting from combined heat and mass buoyancy effects, Int Commun Heat Mass Transfer, 15(1) (1988) 17-30.

6. F.C.Lai and F.A.Kulacki, Non-Darcy mixed convection along a vertical wall in a saturated porous medium, J Heat Transfer, 113 (1991) 252-255.

7. A.Nakayama and M.A.Hossain, An integral treatment for combined heat and mass transfer by natural convection in a porous medium, Int J Heat Mass Transfer, 38(4) (1995) 761-765.

8. K.A.Yih, The effect of transpiration on coupled heat and mass transfer in mixed convection over a vertical plate embedded in a saturated porous medium, Int Commun Heat Mass Transfer, 24(2) (1997) 265-275.

9. A.J.Chamkha, H.S.Takhar and V.M.Soundalgekar, Radiation effects on free convection flow past a semi-infinite vertical plate with mass transfer, Chem. Eng. J, 84(3) (2001) 335-342.

10. P.Ganesan and G.Palani, Natural convection effects on impulsively started inclined plate with heat and mass transfer, Heat Mass Transfer, 39(4) (2003) 277-283.

11. Raghunath and Siva Prasad, Heat and mass transfer on unsteady MHD flow of a visco-elastic fluid past an infinite vertical oscillating porous plate, British Journal of Mathematics \& Computer Science, 17(6) (2016) 1-18.

12. G.M.Oreper and J.Szekely, The effect of an externally imposed magnetic field on buoyancy driven flow in a rectangular cavity, J Cryst Growth, 64(3) (1983) 505-515.

13. M.A.Hossain and A.C.Mandal, Mass transfer effects on the unsteady hydromagnetic free convection flow past an accelerated vertical porous plate, J Phys D Appl Phys, 18(7) (1985) 163-169.

14. M.Veera Krishna and M.Gangadhar Reddy, MHD free convective rotating flow of Visco-elastic fluid past an infinite vertical oscillating porous plate with chemical reaction, IOP Conf. Series: Materials Science and Engineering, 2016. 